\title{
Carnets
}

Revue électronique d'études françaises de l'APEF

Deuxième série - 20 | 2020

Imaginaire(s) du Voyage

\section{Aux détours de la vie d'Augustin Meaulnes et de Gaspard Fontarelle, le devenir de l'Homme}

\section{Maria Eugénia Pereira}

\section{(2) OpenEdition}

1 Journals

Édition électronique

URL : http://journals.openedition.org/carnets/12362

DOI : 10.4000/carnets. 12362

ISSN : 1646-7698

Éditeur

APEF

Référence électronique

Maria Eugénia Pereira, «Aux détours de la vie d'Augustin Meaulnes et de Gaspard Fontarelle, le devenir de l'Homme », Carnets [En ligne], Deuxième série - 20 | 2020, mis en ligne le 30 novembre 2020, consulté le 20 avril 2021. URL : http://journals.openedition.org/carnets/12362 ; DOI : https:// doi.org/10.4000/carnets. 12362

Ce document a été généré automatiquement le 20 avril 2021.

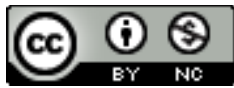

Carnets est mis à disposition selon les termes de la licence Creative Commons - Atribution - Pas d'utilisation commerciale 4.0 International. 


\title{
Aux détours de la vie d'Augustin Meaulnes et de Gaspard Fontarelle, le devenir de l'Homme
}

\author{
Maria Eugénia Pereira
}

1 Il y a des romans qui s'adressent aussi à nous à l'âge mûr, bien qu'ils aient pu avoir été lus lors de notre jeunesse, au moment de la recherche enthousiaste d'un idéal, d'un absolu. C'est le cas du Grand Meaulnes d'Alain-Fournier et du Pays où l'on n'arrive jamais d'André Dhôtel, deux œuvres qui, parce qu'elles disent l'humain, tout l'humain, réconcilient les intermittences de la conscience et les grandes cohérences de l'âge: l'enfance, l'adolescence, l'âge mûr et la vieillesse.

2 Envahis par un esprit d'enfance, les deux auteurs éprouvent une poignante douleur, un profond tourment à supporter le monde, aussi vont-ils vivre par l'écriture de leurs œuvres et pour elles l'aventure d'une quête du sens du monde et de soi. Épris d'errance, vivant dans l'impossibilité d'accepter le monde tel qu'il est, ils se lancent dans la reconstitution du souvenir, dans la reconstruction du passé, dans l'édification d'un présent multidimensionnel où l'être se maintient en attente. Les héros de ces deux œuvres doivent, par conséquent, être interprétés comme la projection de l'individu luimême :

L'individu, en effet, apparaît en proie à des conflits psychologiques qui naturellement varient (plus ou moins selon leur nature respective) avec la civilisation et le type de société auxquels il appartient. De ces conflits, il est le plus souvent inconscient, étant donné qu'ils sont très généralement le fait de la structure sociale elle-même et le résultat de la contrainte qu'elle fait peser sur ses désirs élémentaires. (Caillois, $1938: 24$ )

Conçus comme le témoignage d'une expérience essentielle, d'une conscience au réel, les deux romans cherchent à recomposer l'infini et à éterniser l'homme par le biais du merveilleux: Alain-Fournier nous dit, à ce sujet, vouloir «exprimer le mystère du monde inconnu qu'[il] désire. Et comme ce monde est fait de vieux souvenirs, de vieilles impressions inconscientes, [il] voudrai[t] exprimer le mystère de ces impressions particulières, que le monde [lui] laisse » (Rivière \& Alain-Fournier, $1937: 358)^{1}$; André 
Dhôtel affirme, lui aussi, que «la féerie est présente dans le réel : c'est ce qu'[il a] découvert grâce aux champignons, par exemple. (...) Pour trouver la féerie, il ne faut pas lever la tête vers le ciel, il faut d'abord fouiller le réel : au collège d'Autun, c'étaient les pierres, ensuite ce furent les fleurs, et maintenant ce sont les champignons" (Dhôtel, 1984 : 34). Les deux auteurs mettent donc en place un autre ordre du monde dont nous sommes à la fois les spectateurs et les acteurs et où il nous est donné de retrouver en profondeur le sens de la vie de l'homme.

Orphelin de père, Augustin Meaulnes, devenu le Grand Meaulnes par ses exploits d'élève insoumis, semble incarner les mythes de Phaéton et d'Icare, dans son insistance à prendre l'envol vers des contrées mystérieuses, aux confins inconnus, et dans son désir d'accéder à l'insaisissable.

5 Tout comme Clymène, la mère de Phaéton, Madame Meaulnes, lui laisse prendre l'envol seul et lui ouvre, ainsi, les portes de la découverte de sa véritable identité (cf. Ovide, 1969a : 33-34). Fils lui aussi du «Soleil " ${ }^{2}$, chaque petit événement rapporté par sa mère doit être lu comme un signe de l'illusion et les mots du narrateur sont, eux aussi, porteurs d'un message, qui nous ouvre la voie sur le caractère spécial de Meaulnes: «Elle eût même, dès qu'elle parla de son fils, un air supérieur, et mystérieux qui nous intrigua » (Alain-Fournier, 1972: 19). Nous sommes donc invités à maintenir notre imagination en éveil et à nous laisser transporter hors de la réalité.

Coincé entre l'être, la mère, et le paraître ${ }^{3}$, l'illusion, Meaulnes va chercher à réconcilier ces deux mondes et nous nous attendons à ce qu'il y arrive, dans la mesure où nous avons déjà découvert qu'il possède un caractère fabuleux.

7 Il est rarement fait référence à la paternité d'Augustin et ce vide laisse libre cours à l'imagination du lecteur. Le père, appartenant, lui aussi, à l'être, est loin de la figure mythique du père héroïque. Incapable de transporter son fils dans le paraître, il gère un manque et, en conséquence, le lecteur va chercher à combler cette carence et à lui trouver une filiation divine, bien plus à la hauteur de cet être supérieur et extraordinaire. En cherchant à concilier les deux mondes, Meaulnes n'est-il pas, lui aussi, un mortel insoumis, qui brave le ciel pour réaliser ses rêves : tel Phaéton, « (...) il se dirige à la hâte vers les lieux où se lève son père » (Ovide, 1969a : 34).

8 Tout comme cette figure mythique, Meaulnes est fier, rebelle, téméraire, impulsif, ivre du désir de se lancer dans de hauts prodiges, de vivre dans l'incommensurable et de fuir la banalité de sa vie quotidienne. Nourri donc d'aventures, muni d'illusions, le jeune adolescent fourniérien se lance, tel Phaéton, à la conquête de l'inconnu, «la pensée toute pleine des régions éthérées " (ibid.):

Un pied sur le devant, dressé comme un conducteur de char romain, secouant à deux mains les guides, il lance sa bête à fond de train et disparait en un instant de l'autre côté de la montée. (Alain-Fournier, $1972: 33)^{4}$

9 Ce conducteur de char représente la nature spirituelle de l'homme: Meaulnes, à la recherche de sa propre identité, conduit les chevaux de sa destinée.

10 Fougueux, emporté, comme Icare il se laisse aller par l'immensité de sa démesure et pousse trop loin son désir de voler en liberté. Icare, grisé par l'envol, néglige tous les conseils de son père et monte haut dans le ciel, faisant fondre la cire qui tenait ses ailes ${ }^{5}$. Meaulnes se laisse, lui aussi, enivrer par son désir d'aventures et n'écoute personne. 
11 Gaspard Fontarelle fut abandonné par ses parents et laissé aux soins d'une tante désintéressée de son avenir. Victime de l'austère piétisme inculqué par Gabrielle Berlicaut, Gaspard Fontarelle croit que tous les incidents soufferts pendant son enfance lui ont été infligés pour expier la faute du comportement déviant de ses parents : ces derniers étaient devenus nomades et voyants, alors qu'ils appartenaient à une des plus vieilles familles du pays.

Gaspard souffre d'un non-être qui finit par bouleverser son âme et devient profondément «ignorant des choses du monde» (Dhôtel, 1955: 14). "Vu de l'extérieur ", nous dit Philippe Blondeau, «il reste lui-même un peu extérieur aux événements (...) a priori dépourvu d'épaisseur, presque transparent, il constitue un révélateur exceptionnel de la réalité devant laquelle il semble s'effacer» (2003: 161). Toutefois, bien qu'il veuille passer inaperçu et se garder de rêver à des équipées extraordinaires au sein de Lominval - le cadre de la réalité -, il y est néanmoins initié aux périls d'aventures involontaires.

Comme $\mathbb{C E d i p e}^{6}$, abandonné à la naissance, il vit aveuglément une partie de sa vie, dans l'attente d'une "parole qui lui ferait découvrir tout ce qu'il ignorait» (Dhôtel, 1955 : 18). Mais, contrairement au jeune đEdipe, il connaît ses origines et, par conséquent, il ne peut vivre heureux dans l'insouciance.

Des rêveries avaient néanmoins déjà traversé son esprit et l'avaient entraîné vers des pensées plus consolantes: «Il avait dans l'idée qu'il existait des gens et même des peuples qui n'avaient rien de commun avec l'humanité telle qu'il la connaissait d'après les habitants de Lominval» (ibid. : 17).

Albert Béguin nous explique, ainsi, les raisons du rêve :

Un être tourmenté de pareilles anxiétés, impuissant à leur trouver une solution, poursuivi en même temps par le constant déséquilibre d'une nature instable et prompte à se déprimer, cherchera des moyens de défense contre un univers extérieur qui est tout de menaces. Contre les hommes, mais aussi contre ses propres faiblesses et ses doutes, l'oubli peut être trouvé dans le jeu. Puisque la vie, lorsqu'on l'affronte avec toute la gravité de son interrogation et de ses espoirs, est terrible, il faut se jouer d'elle. (1967: 33)

16 Gaspard Fontarelle va, lui aussi, entrer dans le jeu et se laisser aller au rêve pour fuir sa solitude et son angoisse.

Promeneur solitaire infatigable, Gaspard observe la nature environnante dans l'espoir d'y trouver un regard, « où il y [aurait] comme une fissure éblouissante » (Dhôtel, 1955 : 18). CEdipe s'en est allé consulter les oracles avant de se lancer dans l'aventure, dans la quête de son identité, Gaspard Fontarelle, soucieux de noter les moindres détails d'une échappée extraordinaire, s'est vu reflété dans le regard de l'enfant fugitif et il s'évade, lui aussi, de ce monde privé de splendeurs et de rêves, auquel il n'a jamais véritablement pris part, pour retrouver, lui aussi, son identité.

18 À la ressemblance de l'orphelin Meaulnes, cet enfant abandonné va devenir, lui aussi, un fugueur à la recherche de l'unité, d'un pays sans nom où il pourra se retrouver.

Malmenés par les adultes, aimants ou négligents, les jeunes adolescents d'AlainFournier et d'André Dhôtel suivent une destination qui se trouve au-delà du monde sensible. Les deux personnages de ces romans sont entêtés et orgueilleux, ils agissent donc de façon autonome et spontanée. Mus par des réflexions et des pensées vivantes, ils agissent selon leur désir et leur impulsion et c'est pourquoi ils nous transportent 
dans le magique. L'inassouvissement les rendant malheureux, ils préfèrent se lancer à cœur ouvert dans l'inconnu, dans la découverte d'un monde autre et de soi-même.

En quête d'un absolu, voulant prouver au monde entier qu'ils ne sont pas des êtres communs, Augustin Meaulnes et Gaspard Fontarelle initient, sans le savoir, mais librement, une nouvelle étape de leur vie.

Hors du monde arthurien, la quête du Graal semble avoir perdu toute son efflorescence mythique, toute sa luxuriance médiévale, mais Alain-Fournier et André Dhôtel ont su se l'approprier par le biais de la poésie et lui donner une continuité afin de la rendre moderne. Selon Isabelle Cani :

Le propre de la poésie est sans doute de créer un univers intemporel, affectif et symbolique, qui se rit des pesantes distinctions chronologiques dans lesquelles nous nous mouvons. Le Graal peut donc y côtoyer sans difficulté des images du quotidien. Souvent, la différence entre passé et présent est consciemment refusée : le poète revendique la liberté de se reconnaitre dans son héritage de l'humanité. (1996:174)

2 En somme, en se jetant dans l'inconnu, dans des espaces imaginaires, nos deux héros répètent le mythe, mais en lui donnant l'échappée libre de ses créateurs.

Sans vouloir prétendre que le personnage fourniérien soit l'avatar du personnage médiéval, la quête de Meaulnes semble avoir été tracée selon celle de Perceval ${ }^{7}$. AlainFournier a respecté les degrés initiatiques du mythe médiéval, le «schéma essentiel » (Baudry, 1998: 77) des équipées de Perceval, mais il lui a fallu arracher progressivement son héros au monde réel par une rupture spatio-temporelle pour justifier la présence du Graal : la campagne qu'il traverse est désertique et il ne peut, par conséquent, se renseigner sur son chemin auprès de personne; le sommeil et la nuit lui font perdre tout repère spatial et temporel et permettent, ainsi, que s'effectue le passage d'un monde à l'autre. Le lecteur se voit donc transporté dans un Ailleurs, sans même se l'être fait expliquer, et il ne s'y sent nullement étranger. Un monde, selon Marie Maclean, où la «merveille [est] intégrée à la réalité » (1973:59) et dans lequel nous avançons en toute sérénité, grâce à la potentialité magique du discours narratif. Claude Herzfeld, « Ni le Xvarnah ni le Graal n'ont une forme spécifique » (1999: 133) et le critique se propose de décrire les différentes formes que revêt le Graal dans le Grand Meaulnes, mais nous ne nous attacherons qu'à celle de la femme, puisque c'est par elle que passe la réalisation de tous ses rêves : «Il put imaginer longuement qu'il était dans sa propre maison, marié, un beau soir, et que cet être charmant et inconnu qui jouait du piano, près de lui, c'était sa femme » (Alain-Fournier, 1972 : 71).

Meaulnes a enfin trouvé le Graal : la femme rêvée, la femme-mère, la princesse, la fée et, par conséquent, il ne pourra désormais plus être le même. Il a rencontré la perfection, c'est-à-dire une femme rêvée au milieu d'enfants de rêves et, par conséquent, l'Absolu semble avoir été atteint. Mais il ne faut pas s'y méprendre, «Il a créé son amour, parfait, irréalisable et hermétique, et il préfère le désespoir qui en résulte à tout espoir qui lui viendra d'un amour humain, impur, changeant, en proie aux regrets » (Maclean, 1973: 93). Meaulnes n'a donc fait que côtoyer la perfection, l'ayant perdue avant même de l'avoir conquise et, par conséquent, le terrestre, le quotidien vont reprendre le dessus. Le héros reconnait, qu'au moment de la rencontre avec la jeune fille, archétype d'un avenir impossible, il avait atteint le plus haut état de la « perfection et de la pureté » et que «Dans la mort seulement [il] retrouverait peutêtre la beauté de ce temps-là... » (Alain-Fournier, 1972 : 169). 
La rencontre de Meaulnes avec Yvonne de Galais figure, ainsi, la quête réussie de la connaissance : il aura compris que «l'enfance est un paradis à jamais perdu et, qu'en conséquence, le bonheur est inaccessible " (Pereira, 2005: 510). La tension constante entre l'ici-bas et le là-bas, c'est-à-dire entre la réalité et l'illusion, forment l'unité même de l'œuvre, et Robert Pickering nous montre cela même :

Les ressorts affectifs d'une conscience profondément poétique, à l'écoute de l'intimité la plus raffinée des aspirations, y vont de pair avec une lucidité qui semblerait très divergente, mais qui fonde l'élan des passions en un traitement réaliste nullement éloigné du mouvement de la société ou des enjeux qui en guettent l'évolution. (1999: 76)

Anne Cousseau considère, elle aussi, que la rencontre de Meaulnes avec Yvonne marque la fin du parcours initiatique. Selon le critique, deux pôles indissociables composent le désir amoureux de Meaulnes, la pureté de l'âme et le souvenir de l'enfance, et, par conséquent, l'ambivalence du bonheur, «qui s'offre dans la conscience de son inaccessibilité [,] fonde précisément l'enseignement délivré à Meaulnes» (1999: 61). C'est sans doute pourquoi Christian Chelebourg estime, lui aussi, que «L'écriture du Grand Meaulnes est le contraire d'une écriture intellectuelle. Elle naît d'un renoncement à l'abstraction » (1999: 34) et que "C'est, pour ainsi dire, en assassinant l'enfance qu'elle en nourrit la nostalgie » (ibid.). Désormais, et parce que la force de la réalité reprend le dessus, la diégèse se trouve envahie de regrets, de faute, de trahison et de mort, comme autant de sillages poétiques laissés derrière le héros.

Le récit dhôtelien repose lui aussi sur l'aventure d'une quête et, tout comme pour le héros fourniérien, c'est un cheval qui va le transporter dans un autre monde, dans un «monde d'originaux » (Dhôtel, $1955: 54$ ).

Loin de Lominval, de cet endroit fermé qui le maintenait dans l'ignorance et la méconnaissance, d'où il est parti « sans le faire exprès » (ibid. : 63), Gaspard s'aventure dans des contrées inconnues - les Ardennes belges, des villes de Belgique, les Bermudes -, d'abord à la recherche d'un enfant fugueur, puis en s'alliant à lui dans la quête du "grand pays".

Le parcours initiatique se fera au rythme des découvertes de Gaspard, le monde se dévoilant progressivement à ses yeux, et chaque exploration faisant partie d'un processus d'apprentissage auquel le mystérieux enfant fugueur d'Anvers se trouve lié.

31 Sorti de Lominval, quittée l'inexpérience de sa vie passée, il découvre ce que ses yeux n'ont pas su voir : le jeune fugueur est, en fait, une fille qui s'appelle Hélène. Mais, à la ressemblance de Meaulnes, un rêve prémonitoire ne s'était-il pas déjà chargé de le lui annoncer?:

Gaspard remarquait [dans son rêve] surtout une maison à quarante étages au sommet de laquelle une jeune fille se promenait avec une ombrelle. À chaque oscillation de la bâtisse, la jeune fille manquait de tomber. Soudain il y eut un mouvement plus brutal, et toutes les pierres se disjoignirent. La jeune fille disparut au milieu des pierres qui roulèrent dans les avenues et formèrent une sorte de montagne mouvante qui allait s'écrouler sur Gaspard quand il ouvrit les yeux. (Ibid. : 98-99)

Hélène porte le bleu le ciel dans les yeux et les étoiles dans les cheveux. Par conséquent, elle n'est pas du monde ci-bas, elle appartient, elle aussi, à un monde supérieur : c'est une sauvage qui récuse le quotidien et la banalité, qui est avide d'évasion, d'aventures, et qui veut poursuivre sa quête du "grand pays » (ibid. : 115) de Jenny, un lieu irréel, bizarre, au paysage composite : «Une drôle de campagne. Des chênes, des bouleaux et 
en même temps des palmiers. Une forêt avec une clairière. Un peu plus loin, on apercevait une mer bleue (...) une file de pommiers sur une terre noire» (ibid.: 114-115). Hélène recherche donc un nulle part et un ailleurs, plusieurs réalités et un rêve, en somme, un non lieu qui n'existe que par le fait qu'il est le "grand pays » de Jenny. Comme nous le dit Michèle Monballin, «Le 'grand pays' recherché par Hélène est conjoint à l'image de sa mère » (1998: 231) et, par conséquent, « il se trouve pourvu d'une valeur affective (...) et d'une valeur symbolique » (ibid.).

L'insolite du paysage, le rend invraisemblable aux yeux des adultes, qui n'ont ni la foi, ni la pureté de l'enfance pour croire à l'existence d'un tel pays. Selon eux, il s'agit d'un délire, d'un pays imaginaire et introuvable, mais, comme le dit l'un des personnages : c'est une «race de jeunes singes qui veulent décrocher le paradis » (Dhôtel, 1955 : 131) et ils vont payer cher le prix d'un rêve.

Ces adultes ont renoncé à l'ambition de rêver, ils pensent avoir trouvé la suffisance et se sont enfermés en un point quelconque de leur existence, se laissant vivre par ce qui n'est, en fait, que de l'insuffisance.

Le seul adulte qui croit en ce pays est un vieux musicien nomade, qui voyage en compagnie de ses deux enfants, eux aussi musiciens. Gaston Bachelard en explique ainsi les raisons :

On dira qu'on voyage pour voir; mais comment bien voir sans s'émerveiller et comment s'émerveiller devant les nouveautés du réel sans un long préambule de rêveries familières? Les grands voyageurs sont d'abord, en une longue adolescence, de grands rêveurs. Pour aimer partir, il faut savoir se détacher de la vie quotidienne. Le goût des voyages relève du goût d'imaginer. Il semble qu'une frange d'imaginaire soit toujours nécessaire pour donner un intérêt aux spectacles nouveaux. (1978: 135)

Niklaas, le vieux musicien nomade, est le seul capable de parler du réel, de susciter des rêves et de voir dans le pays inconnu les forces de l'imaginaire d'Hélène: «je peux avouer que j'ai certaines idées sur le pays d'Hélène. (...) Je connais les lieux cependant où il est possible de voir dans un même paysage certaines espèces de palmiers, des bouleaux, des chênes et peut-être la mer. Il faut tenir compte aussi des illusions d'une enfant » (Dhôtel, 1955 : 149). Préfigurant, déjà, le Graal, Niklaas possède la connaissance $\mathrm{du}$ nomade et, par conséquent, il fait comprendre à Gaspard comment, en parlant du réel, on peut susciter des rêves :

Si tu veux découvrir ce que tu cherches, Gaspard, tu dois tâcher de lire les signes qu'il y a dans les choses. (...) Le pays d'Hélène t'apparaîtra peut-être dans un de ces lieux inconnus dont il y a des milliers de contrées. (...) Un indice en amène un autre, dit Niklaas. La terre est immense, mais il y a des liens entre les choses. (Ibid. : 153)

Niklaas est l'un de ces hommes dignes dont parle Alain Chareyre Méjan ${ }^{8}$ puisque, n'ayant plus rien à perdre, il prétend à tout dans une liberté fabuleuse. Capable donc de capter ce qui, pour l'homme ordinaire, est insaisissable, il se rend disponible à Gaspard pour l'aider à découvrir le Graal. Mais ce dernier, ébloui par la beauté, la splendeur, le mystère des paysages, reste inattentif aux signes, ne tient pas compte des liens entre les choses et finit par oublier que rechercher le "grand pays " c'est aussi rechercher maman Jenny. Il se laisse aller à son expérience de la découverte de la beauté, et croit avoir trouvé le «pays» d'Hélène dans la «splendeur» (Dhôtel, 1955: 161) qu'il entrevoit :

Non loin de l'endroit où ils étaient parvenus, la pièce d'eau s'élargissait en une sorte de baie. Cette baie était encastrée par des chênes et des bouleaux et baignait une 
vaste grève autour de laquelle s'alignaient, dans des caisses, des orangers et des palmiers. Derrière les palmiers s'élevaient les bâtiments d'un énorme château. (Ibid. : 160)

En entrant dans le château, il plonge dans le merveilleux, dans un monde étrange et tout semble être là pour l'égarer encore plus et pour lui faire croire qu'il s'agit du pays d'Hélène.

La pensée de Gaspard tend à vouloir croire qu'il s'agit du " pays » d'Hélène, bien qu'il sache qu'il manque, encore, à cet endroit un élément : «la terre noire » (ibid.: 166). L'esprit envahi et possédé par l'insolite et la fantaisie du lieu et des personnages, Gaspard finit par s'abandonner aux coïncidences extraordinaires qui lui sont présentées et part pour, par la suite, y ramener Hélène.

Hormis sa foi, son désir à vouloir aider son amie, une chose l'intrigue : le départ de ce pays méconnu, la fugue avec Hélène et le chemin du retour se réalisent sans aucune difficulté, ce qui met l'intelligence du jeune garçon en éveil et le mène à considérer que «Tout cela n'est pas normal (...)» (ibid. : 175). Par ailleurs, le narrateur intervient, lui aussi, pour nous faire comprendre que les deux enfants peuvent se trouver sur une fausse piste : «Tout fut inexplicablement facile » (ibid. : 177). Le seul à ne pas croire à la découverte, c'est, précisément, Niklaas : empreint de rêves, se trouvant au plus haut de la dignité de l'homme adulte, il sait que le pays d'Hélène devrait être encore plus beau.

Gaspard, lui aussi muni du don extraordinaire de percevoir les moindres détails, revient sur ce faux-bond et re-déclenche la quête. Une fois de plus, la découverte de la jeune fille s'avère difficile et il tombe dans le théâtre même des initiations : dans un labyrinthe composé d'escaliers et de portes essentiellement closes, mais où chaque porte ouverte débouche sur des personnages insolites, sinon même étranges 9 . Prisonnier de son incroyance, du fait même d'avoir abandonné sa véritable quête, le héros plonge dans le côté obscur d'une même réalité : le château qui, jusque là lui avait apporté tout espoir - de réaliser le rêve d'Hélène - devient, maintenant, le lieu de toutes les horreurs, puisqu'il fait sombrer dans la noirceur de la réalité.

Mais, comme lors de sa vie routinière à Lominval, le cheval pie, le Pégase ${ }^{10}$, réapparaît pour lui faire reprendre vie et lui redonner le désir de parcourir les contrées. Tout comme Meaulnes, ayant perdu tout espoir, une fois la faute commise d'avoir abandonné ses rêves, Gaspard veut réparer sa faute et rendre à Hélène le feu qui brillait dans ses yeux.

Ce que Gaspard et Hélène avaient cru être le fruit du hasard, du caprice - la course vertigineuse du cheval pie vers un on ne sait où - n'était en fait que la force du destin en action. Capable d'entreprendre les plus hauts faits, les plus grandes expéditions, ce Pégase dhôtelien est, comme nous le dit Claude Herzfeld, «l'archétype de l'esprit (...) sous la forme d'un animal enchanté, secourable, au comportement humain, qui manifeste une intelligence et un savoir supérieurs à ceux des hommes » (1998:58). recherche de l'aide de Niklaas, de ce nomade dont on "[espère] bien des choses " (Dhôtel, 1955 : 200). Aux côtés de ces deux êtres de rêve, Gaspard ne peut dorénavant plus échouer ; la quête est relancée, et rien ne doit, désormais plus, entraver la réussite du rêve.

Les catastrophes provoquées par Gaspard à Lominval vont se reproduire et il va, enfin, comprendre, qu'elles n'apportent aucun mal et qu'elles constituent, au contraire, le 
passage pour la réalisation même de ses rêves. Nous avons, à la ressemblance du héros, su décrypter tous les symboles et, par conséquent, une phrase du narrateur - «Mais écoutez ce qui arriva » (ibid. : 225) - nous met en état d'alerte et nous fait comprendre que l'initiation est près d'être finie.

Tout comme dans Le Grand Meaulnes, le cheval transporte ses voyageurs épris de rêves et d'aventure - Niklaas, ses deux enfants, Gaspard et Hélène - vers un domaine méconnu, vers un pays impossible à atteindre, un pays sans nom. Espace et temps ${ }^{11}$ se conjuguent dans l'ineffable pour perdre les personnages ou plutôt pour les conduire vers le Graal : « Ainsi cette course discordante prenait l'allure d'un rite insensé » (ibid. : 239), celui de la découverte.

Dans Le Pays où l'on n'arrive jamais, le Graal «se dissimule sous l'incognito » (Baudry, 1998 : 197) d'autres noms et il prend, ici, le nom de «Maman Jenny " (Dhôtel, 1955 : 240). Une femme, dont le caractère singulier, mais connu de Gaspard, dénonce la valeur de réceptacle et de miracle du Graal :

Une femme, jeune encore, au beau visage, aux lourds cheveux blonds, attendait la clientèle. La femme avait des regards patients et simples. Dans ses yeux bleus néanmoins brillait par instants cette même flamme dure qui avait surpris Gaspard quand il l'avait vue dans les yeux d'Hélène pour la première fois. (Ibid. : 240-241)

La mère d'Hélène a été retrouvée et sa maison, «la caravane» (ibid.: 242), est le « doublet microcosmique du corps matériel et [du] corps mental» (Durand, 1992: 277) de celle-ci et elle est, pour Hélène, le giron maternel tant recherché. En effet, et bien que ce soit une caravane, une tente, elle est l'image de l'intimité qui reposera pour toujours l'esprit de la jeune fille. Cette dernière est parvenue à « l'espace bienheureux, [au] centre paradisiaque " (ibid. : 280), puisqu'elle y a trouvé son identité. Le souvenir qu'Hélène gardait du paysage de son enfance n'a, en fait, pas résisté à la pulsion de la mémoire affective et elle apprend par sa mère que le "grand pays " n'est en fait que toutes les régions par lesquelles, comme nomades, elles étaient passées. Il peut donc être trouvé partout et nulle part. Il est, en fait, le symbole du mode de vie des nomades. Par la force de son désir, Hélène est parvenue à remonter à son origine et à retrouver le monde extraordinaire de l'évasion et de l'aventure.

Or, Gaspard n'est-il pas issu de ce même milieu? N'est-il pas fils de forains lui-même? N'a-t-il pas participé à la quête d'Hélène en la faisant sienne ? De fait, la merveille ne se serait accomplie si Gaspard n'avait eu, lui-même, son Graal, sa fin promise par les différentes catastrophes de son enfance. Ces dernières le liaient déjà à une autre réalité, le plaçaient hors du monde palpable, mais, empêché, par sa tante, d'y voir un signe, il lui fallait quelqu'un d'extraordinaire pour lui « faire découvrir tout ce qu'il ignorait » (Dhôtel, 1955 : 17). Il finit par retrouver ses parents, et par conséquent, son identité aux côtés d'Hélène et, ainsi, par s'unir définitivement à elle. La quête est enfin réussie et la véritable merveille du Graal se trouve dans la découverte, par Gaspard, qu'Hélène est, en fait, son pays et qu' « ils resteraient unis toute leur vie » (ibid. : 249).

Ces deux œuvres romanesques nous renvoient, dans les deux cas, à l'étrange, à l'inconnu pour exprimer le désir de revenir dans le passé, au commencement de l'absolu. Meaulnes et Gaspard s'acharnent à recommencer leur histoire, profondément marqués par la nostalgie de l'enfance, et veulent atteindre le paradis, un paradis au caractère paradoxal de Mircea Eliade : «d'une part, il représente le contraire de ce monde-ci : pureté, liberté, béatitude, immortalité, etc. ; d'autre part, il est concret - i.e il n'est pas 'spirituel' - et fait partie de ce monde, puisqu'il a une réalité et une identité 
géographiques » $(1971: 197)$. Le « domaine perdu » et le " grand pays » sont ce paradis, un endroit ou chaque héros retrouve sa plénitude spirituelle, un espace idyllique, mais proche de la réalité palpable, c'est-à-dire, où ils retrouvent des fragments de paysages connus. Parce qu'ils sont imprégnés d'une atmosphère étrange ${ }^{12}$ - comme dans Le Grand Meaulnes - ou parce qu'ils constituent un tout hétéroclite - comme dans Le Pays où l'on n'arrive jamais, ces paysages se rapprochent aussi, dans une certaine mesure, de l'Eden primordial.

51 Mais le chemin vers le paradis, c'est-à-dire le passage du monde-ci au monde-là, joue, selon Mircea Eliade, un rôle essentiel: «c'est lui qui reçoit les indications d'ordre surnaturel qui lui permettent de guider [les héros] dans [leur] prodigieux vagabondage " (1971: 202). Comme nous l'avons vu auparavant, sur les chemins parcourus par Meaulnes et Gaspard se réalise l'initiation, c'est pourquoi ils sont jalonnés, de part et d'autre, par la nature et ils sont parsemés d'obstacles qui rendent la recherche difficile. Transformés rituellement en labyrinthes, y pénétrer équivaut «à un retour mystique à la Mère " (Eliade, 1989: 211) et les adolescents ne sont-ils pas en quête d'une femme-mère qui les protègera contre les méfaits du monde adulte? Meaulnes ne voit-il pas en Yvonne la mère protectrice, capable de tout pour apaiser ses tourments?: "'Et j'essayais de le consoler, de le rassurer. Rien ne calmait son angoisse'. Alors j'ai dit : 'S'il faut que vous partiez, si je suis venue vers vous au moment où rien ne pouvait vous rendre heureux, s'il faut que vous m'abandonniez un temps pour ensuite revenir apaisé prés de moi, c'est moi qui vous demande de partir...'" (Alain-Fournier, 1972 : 206) ; Gaspard n'éprouve-t-il pas, lorsque sa mère le serre dans ses bras, qu'une vie neuve s'ouvre devant lui ? (Cf. Dhôtel, $1955: 248$ ).

Mircea Eliade nous dit que "L'homme a besoin d'un 'chemin' pour (...) achever sa destinée » (1971 : 202) et nos deux héros l'ont trouvé, involontairement, soit, mais ils le suivent sans jamais revenir sur leurs pas, sans jamais renier leurs rêves. En fuite devant l'approximation imminente de l'âge adulte, devant le désespoir provoqué par la perte de la pureté de l'enfance, munis d'une profonde foi en eux-mêmes, d'une vaste autoconfiance, ils se lancent sur les chemins d'un paradis. L'homme possédant cette foi des deux rêveurs peut, lui aussi, transformer ce monde-ci, le monde réel, et y trouver son paradis intérieur et extérieur.

53 La foi en soi aide l'homme à surmonter ses problèmes, à vaincre ses défis, à se déjouer d'un destin banal et sans espoir et à souffrir "une mutation ontologique du régime existentiel » (ibid. : 206). Meaulnes et Gaspard ont été créés à l'exemple de l'homme et, par conséquent, ils prennent vie sous les yeux du lecteur et s'acharnent à surmonter tous les obstacles rencontrés lors de leur initiation pour devenir heureux, bien qu'ils n'y parviennent véritablement pas. Mircea Eliade nous explique, philosophiquement parlant, ainsi l'initiation: "À la fin des épreuves, le néophyte jouit d'une tout autre existence qu'avant l'initiation: il est devenu autre » (ibid.). Le critique continue son explication sur les trois grandes catégories d'initiation, mais nous n'allons nous intéresser qu'à la première, puisque nous estimons que l'initiation de nos deux jeunes héros appartient à celle-ci :

$1^{\circ}$ La première catégorie comprend les rituels collectifs par lesquels s'effectue le passage de l'adolescence à l'âge adulte, et qui sont obligatoires pour tous les membres de la communauté. La littérature ethnologique désigne ces cérémonies par les termes «rites de puberté ", «initiation tribale » ou «initiation de classe d'âge ». Les autres initiations se distinguent de celles de puberté en ce qu'elles ne 
sont pas obligatoires pour tous les membres de la communauté et que la plupart se pratiquent individuellement ou par groupes assez restreints. (Ibid. : 207)

Meaulnes et Gaspard sont des adolescents qui refusent de grandir, de devenir adultes, d'abandonner leurs rêves et, par conséquent, ils sont contraints à établir une relation entre l'enfance et l'état adulte, à vivre avec leur sexualité, à contrôler leur rêve, en somme, à dépasser " le mode naturel ", propre à l'enfance, et à accéder au "mode culturel », celui des valeurs spirituelles ${ }^{13}$. Devenir adulte ne veut pas dire être accroché au monde matériel et, par conséquent, l'initié est préparé à devenir un homme spirituel, qui connaît le sens profond de l'existence. Par l'initiation, le jeune se métamorphose en un autre, proche du transcendantal, capable d'unifier le pluriel de l'expérience interne.

5 Trouvée leur personnalité, reconnue leur identité, la découverte de la Connaissance prend la forme d'un éternel retour puisque les deux protagonistes sont allés puiser à leur enfance pour découvrir la Vérité et fuir leur destin. Mais, selon la vision de Georges Bataille :

UN HOMME EST UNE PARTICULE INSÉRÉE DANS DES ENSEMBLES INSTABLES ET ENCHEVÊTRÉS. Ces ensembles composent avec la vie personnelle à laquelle ils apportent des possibilités multiples [...]. A partir de la connaissance, l'existence d'une personne n'est isolée de celle de l'ensemble que d'un point de vue étroit et négligeable (1954:100).

Nos deux personnages excentriques vivent dans l'insuffisance d'une existence banale et ils veulent changer leur condition, ils veulent combattre cette nonchalance, avancer dans l'expérience intérieure, la « souveraine conscience de soi » (Bataille, 1954).

L'être humain désoeuvré cherche un guide de créativité spirituelle et la littérature peut, par la poétique, être ce guide. Par l'intensité du rêve et par l'habileté du récit, le Grand Meaulnes et Le Pays où l'on n'arrive jamais concilient l'objet même de la quête de l'homme : la recherche d'un Nulle part et d'un Ailleurs, d'un monde-ci et d'un mondelà, de la réalité palpable et de la réalité sensible.

8 Le principe d'insuffisance qui gère toute vie humaine pousse l'être à chercher dans l'art ce qu'il ne peut trouver dans le quotidien: poussé par le désir d'un renouvellement total, il se laisse guider par l'inconscient et entraîner dans l'univers du rêve et de l'imaginaire. L'inquiétude devant la solitude déclenche un profond besoin d'évasion et de rêve qui peut transmuer l'existence. Par les arts et la littérature, l'être individuel se joue de sa destinée, se moque d'elle et y échappe ne serait-ce que le temps de quelques lettres ou de quelques phrases écrites, de quelques coups de pinceaux, de quelques notes de musique. Il s'évade et en s'évadant il oublie son destin tragique.

Phaéton, Icare, ๔Edipe sont restés des mythes, le Grand Meaulnes, Gaspard Fontarelle les ont fait revivre par le biais d'une réincarnation modernisante. Tous, en fait, ne sont là que pour dire l'homme, pour l'écrire dans toute sa dimension plurielle, pour l'éterniser. " La vie ", nous dit Georges Bataille, "va se perdre dans la mort, les fleuves dans la mer, et le connu dans l'inconnu. La connaissance est l'accès à l'inconnu » (1954: 119). 


\section{BIBLIOGRAPHIE}

ALAIN-FOURNIER (1972). Le Grand Meaulnes. Paris : Gallimard/Librairie Arthème Fayard et Emile-

Paul.

BACHELARD, Gaston (1978). Le droit de rêver. Paris : PUF.

BATAILLE, Georges (1954). L'expérience intérieure. Paris : Gallimard.

BAUDRY, Robert (1998). Graal et littératures d'aujourd'hui. Rennes : Terre Brume.

BÉGUIN, Albert (1967). L'âme romantique et le rêve. Essai sur le romantisme allemand et la poésie

française. Paris : José Corti.

BLONDEAU, Philippe (2003). André Dhôtel ou les merveilles du romanesque. Paris : L'Harmattan.

CAILLoIS, Roger (1938). Le Mythe et l'homme. Paris : Gallimard.

CANI, Isabelle (1996). « Le Graal aujourd'hui : pour une typologie des œuvres », Graal et Modernité,

Actes du Colloque de Cerisy. Paris : Dervy (Cahiers de l'Hermétisme), pp. 169-181.

CHelebourg, Christian (1999). « Poétique du désir frustré : Le Grand Meaulnes et la femme

imaginaire ", in Alain Buisine et Claude Herzfeld (orgs.). Mystères d'Alain-Fournier, Actes du

Colloque de Cerisy. Paris : Nizet, pp. 29-50.

COUSSEAU, Anne (1999). «L'enfance perdue comme figure du bonheur », in Alain Buisine et Claude Herzfeld (orgs.). Mystères d'Alain-Fournier, Actes du Colloque de Cerisy. Paris : Nizet, pp. 51-64.

DHÔTEL, André (1984). L'Ecole buissonnière : Entretiens avec Jérôme Garcin. Paris : Pierre Horay.

DHôTEL, André (1955). Le Pays où l'on n'arrive jamais. Paris : Pierre Horay.

DURAND, Gilbert (1992). Les Structures anthropologiques de l'imaginaire. Paris : Dunod.

ELIADE, Mircea (1989). Mythes, rêves et mystères. Paris : Gallimard.

ELIADE, Mircea (1971). La Nostalgie des origines. Paris : Gallimard.

GUÉNO, Jean-Pierre \& RIVIÈRE, Alain (1995). La Mémoire du Grand Meaulnes. Paris : Robert Laffont.

HERZFELD, Claude (1999). « Lecture Mythique du Grand Meaulnes », in Alain Buisine et Claude Herzfeld (orgs.). Mystères d'Alain-Fournier, Actes du Colloque de Cerisy. Paris : Nizet, pp. 125-138.

HERZFELD, Claude « Manifestation de l'esprit dans Le pays où l'on n'arrive jamais », André Dhôtel, Actes du Colloque d'Angers des 6 et 7 décembre 1996. Angers : Presses de l'Université d'Angers, pp. 57-71.

JOUET, Serge (1983). «Le Sang d'un poète et les surréalistes », in Jean Cocteau : Le Testament d'Orphée, Le Sang d'un poète. Monaco : Editions du Rocher, pp. 11-13.

MACLEAN, Marie (1973). Le Jeu suprême. Paris : Corti.

MÉJAN, Alain Chareyre (1998). André Dhôtel, Actes du Colloque d'Angers des 6 et 7 décembre 1996. Angers : Presses de l'Université d'Angers, pp. 73-78.

MONBALLIN, Michèle (1998). « Le lieu perdu / inatteignable dans Le Grand Meaulnes et Le Pays où l'on n'arrive jamais : pour une interrogation sur le 'réalisme magique'" . André Dhôtel, Actes du Colloque d'Angers des 6 et 7 décembre 1996. Angers : Presses de l'Université d'Angers, pp. 223-241. 
oVIDE (1969a). Les Métamorphoses, Tome I, Livre I. Trad. Georges Lafaye. Paris : Les Belles Lettres. oVIDE (1969b). Les Métamorphoses, Tome I, Livre II. Trad. Georges Lafaye. Paris : Les Belles Lettres. PEREIRA, Maria Eugénia Tavares (2005). Le Réalisme Magique : une dimension des Lettres Françaises du $x x^{e}$ siècle, Thèse de Doctorat. Aveiro : Universidade de Aveiro.

PICKERING, Robert (1999). « Pays perdu, bonheur manqué, joie étrange : Alain-Fournier et les enjeux d'une civilisation mortelle ». Revue d'Histoire Littéraire de la France, oํ 1, pp. 75-94.

RIVIÈRE, Jacques \& Alain-Fournier (1937). Correspondance 1905-1914, Tome II. Paris : Gallimard. SOPHOCLE (1994). CEdipe Roi, Tome II. Trad. Paul Mazon. Paris : Les Belles Lettres.

\section{NOTES}

1. Lettre du samedi matin du 15 décembre 1906.

2. Père de Phaéton.

3. Termes que nous avons empruntés à Marie Maclean, dans Le Jeu suprême (1973: 13).

4. (cf. Ovide, 1969b: 42).

5. (cf. ibid. : 67-68).

6. Nous nous sommes inspirés de la version de la légende de Sophocle (1994).

7. Cf. les différentes étapes établies du parcours initiatique de Meaulnes par Pierre Baudry (ibid.).

8. L'homme digne, nous dit-il dans ces pages, « est doué d'une intelligence singulière centrée autour d'une logique de rêve qui le confronte à des connaissances soi-disant infaillibles", «D'honorables insouciants» (Méjan, $1998: 76)$.

9. La logique de cet épisode est la même que celle du film Le Sang d'un poète de Jean Cocteau : c'est le rêve qui conduit le héros dans cet univers onirique, où « chaque chambre semble être habitée par un souvenir » (Jouet, 1983 : p. 13). Rêveur à défaut, Gaspard s'effraie avec les personnages en cire, les masques, les mannequins sans tête, des figures de pierre et de marbre qui peuplent cet autre côté du château et qui semblent le suivre du regard, comme s'ils représentaient une descente du héros en soi-même. La faute commise, l'abandon de la recherche du véritable Graal, ne peut que le conduire au néant, à l'immobilité de sa vie passée, en somme, à la réalité.

10. Nous découvrons, à la fin du roman, que le cheval pie avait appartenu à " Maman Jenny », et qu'elle l'avait vendu. Mais, « possédé par un feu qui n'est pas de ce monde » (Dhôtel, 1955 : 242), gouverné par des " yeux de rêve » (ibid.), il s'enfuyait de chez son nouveau maître pour revenir chez la nomade.

11. Le paysage, tout comme dans l'œuvre d'Alain-Fournier, est désertique et les chemins, étroits et mal empierrés, au détour impossible, conduisent inévitablement «à une région inconnue des hommes» (André Dhôtel, 1955 : p. 237). Le temps est flou, imprécis, comme pour brouiller le héros, pour participer à son égarement.

12. «Un grand silence régnait sur les berges prochaines. Le bateau filait avec un bruit calme de machine et d'eau. On eût pu se croire au cœur de l'été. On allait aborder, semblait-il, dans le jardin de quelque maison de campagne. » (Alain-Fournier, $1972: 75$ ).

13. Termes repris de Mircea Eliade dans La Nostalgie des origines (1971). 


\section{RÉSUMÉS}

Les héros du Grand Meaulnes et du Pays où l'on n'arrive jamais sont envahis par un esprit d'enfance et ne supportent donc pas le monde tel qu'il se présente. En prise à une douleur profonde, ils prennent l'envol vers des contrées mystérieuses et inconnues, dans leur désir d'accéder à l'insaisissable. Alain-Fournier et André Dhôtel nous invitent donc à découvrir un monde autre, où le magique est intégré à la réalité. Nous y pénétrons sans aucun questionnement, en acceptant l'insolite et en nous détachant de la vie quotidienne et du banal par le biais de l'imagination qui nous est restée de l'enfance.

The heroes of Grand Meaulnes and of Pays où l'on n'arrive jamais are invaded by a spirit of childhood and therefore cannot stand the world as it presents itself. In a deep angst, they through themselves into mysterious and unknown lands in their desire to access the elusive. AlainFournier and André Dhôtel therefore invite us to discover a different world, where magic is integrated into reality. We enter it without questioning, accepting the unusual and detaching ourselves from everyday life and the mundane through the childhood imagination that has remained with us.

\section{INDEX}

Mots-clés : Fournier (Alain), Le Grand Meaulnes, André Dhôtel, Le Pays où l'on n'arrive jamais, errance

Keywords : Fournier (Alain), Le Grand Meaulnes, André Dhôtel, Le Pays où l'on n'arrive jamais, wandering

\section{AUTEUR}

\section{MARIA EUGÉNIA PEREIRA}

(Universidade de Aveiro - Portugal)

epereira[at]ua.pt 